\title{
The Effect of Resilience Training on Quality of Work Life (QoWL) and Job Stress of Nurses in Emergency Department of Shahid Mohammadi Hospital in Bandar Abbas in 2018
}

\author{
Meysam Arbuzia ${ }^{1}$, Mozhgan Rahnama², Abdolghani Abdollahimohammad ${ }^{3}$, Hamideh Goli, Majid Reza Akbarizadeh ${ }^{5}$ \\ Mahin Naderifar 6 \\ ${ }^{1}$ MSc. Student, Nursing Department, Faculty of Nursing and Midwifery, Zabol University of Medical Sciences, Iran. \\ ${ }^{2}$ Department of Nursing, Faculty of Nursing and Midwifery, Zabol University of Medical Sciences, Zabol, Iran. \\ ${ }^{3}$ Department of Nursing, Faculty of Nursing and Midwifery, Zabol University of Medical Sciences, Zabol, Iran , \\ ${ }^{4}$ Department of Paediatrics, Faculty of Nursing, Sabzevar University of Medical Sciences, Sabzevar, Iran. ${ }^{5}$ Department \\ of Paediatrics, Faculty of Medicine, Zabol university of Medical Sciences, Zabol, Iran. ${ }^{6}$ Department of Nursing, Faculty \\ of Nursing and Midwifery, Zabol University of Medical Sciences, Zabol, Iran.
}

\section{ABSTRACT}

\section{BACKGROUND}

Nurses face many stressful situations due to their professional nature which affect their quality of work life (QoWL). The aim of this study was to determine the effect of resilience training on QoWL and job stress of nurses.

\section{METHODS}

This quasi-experimental pre-test - post-test design was performed on 30 nurses working in the Emergency Department of Shahid Mohammadi Hospital, who were divided into intervention and control groups $(n=15)$. The intervention group received 8 sessions of 30-45-minute resilience training in two months and the control group did not receive any intervention during this period. QoWL and job stress were assessed by Walton Quality of Work Life questionnaire and Gray-Toft and Anderson's Nursing Stress Scale (NSS) in both groups before and 1 month after intervention, respectively. Statistical analysis was performed using SPSS ver. 22, descriptive statistics, RM-ANOVA test, and Bonferroni ad-hoc test.

\section{RESULTS}

The findings of this research showed no statistically significant difference between the intervention and control groups after the intervention in terms of QoWL except social integration, total working environment, and constitutionalism in the organization ( $p>0.05)$. Also, there was no statistically significant difference between the intervention and control groups after the intervention in terms of job stress $(\mathrm{p}>0.05)$.

\section{CONCLUSIONS}

The results of this study show that resilience training can be used to improve the QoWL indicators of social integration, the total life space, and constitutionalism in the organization.
Corresponding Author:

Mahin Naderifar,

Department of Nursing,

Faculty of Nursing and Midwifery,

Zabol University of Medical Sciences,

Zabol, Iran.

E-mail:mahin.naderifar@gmail.com

DOI: 10.14260/jemds/2019/685

Financial or Other Competing Interests: None.

How to Cite This Article:

Arbuzia M, Rahnama M, Abdollahimohammad A, et al. The effect of resilience training on quality of work life (QoWL) and job stress of nurses in emergency department of shahid mohammadi hospital in Bandar Abbas in 2018. J. Evolution Med. Dent. Sci. 2019;8(42):3161-3165, 10.14260/jemds/2019/685

Submission 21-08-2019, Peer Review 01-10-2019, Acceptance 08-10-2019, Published 21-10-2019.

\section{KEY WORDS}

Resilience, Quality of Work Life, Job Stress, Nurses, Training 


\section{BACKGROUND}

Nurses are one of the main links in the healthcare chain, besides caring for the patient in various physical, mental, spiritual, and other areas, also play a role of a parent, spouse, child, student, etc. in their personal life. If nurses play these roles simultaneously, they will endanger their mental health.(1) Nurses make up the largest number of human resources in hospitals, and inadequate productivity of this skilled human resources limits service delivery or increases the cost of providing health services.

Emergency department (ED) nurses, in addition to enduring the pervasive mental and physical pressures in all wards of the hospital, face certain stresses such as dealing with the critical condition of the patient and the time urgency, which in turn causes negative emotions.(2) Quality of life (QOL) is a comprehensive concept that encompasses various aspects of life such as financial status, occupation, love, religion, as well as physical, mental, and social health,(3) but QoWL is a comprehensive program that enhances employee satisfaction, enhances learning in the environment, and helps them manage, change, and transform.(4) Etebari Aslet al. showed in their study that $83.7 \%$ of nurses had a moderate QoWL and only $4.9 \%$ of them reported an optimal QoWL.(5)

Stress is one of the factors affecting the QoWL.(6) Stress reduces the quantity and quality of work life.(7) In this regard, Khaghanizadeh et al. reported that nurses with higher QoWL experience less job stress. ${ }^{\left({ }^{8}\right)}$ Stress is an inevitable part of life that can turn into a disease or a positive experience, depending on the stress and the quality of one's adaptation. ${ }^{(9)}$ One of the stressful situations for most people is their workplace conditions, which are called job stressors.(10) Job stress occurs when there is an imbalance between the demands (Expectations) in the workplace and the ability of the employees ${ }^{(8)}$ and can also have negative consequences on the performance of individuals and ultimately the organization in the long run.(10) Nursing is stressful and highrisk occupation. Workloads, excessive workload, inadequate time, staff shortages, excessive working hours, irregular shift work, conflict with other colleagues and high job demands have reported as the stressors has of this profession.(8) Hosseini et al. stated in a study that job stress in ED nurses was significantly higher than in other ward nurses. (11)

Results of one study demonstrated a statistically significant relationship between QOWL and QOL.(12) Poor QOL leads to weakened spirit, low sense of cooperation and responsibility, increased physical pain, anxiety and depression, and ultimately poor performance, which in turn can affect one's QoWL.(13)Resilience promotion is one of the psychological interventions that can improve nurses' QOL. (14) Mannix et al. concluded that resilience and optimism training improve nurses' QOL and self-efficacy.(15) The results of the Barghi Irani et al. also showed that resilience training improves the QoWL of nurses.(14)

Basically, the methods used by individuals to moderate their stress levels is a factor that help them cope with stress. These efficient methods are based on resilience.(9) Connor et al. colleagues showed in a research that resilience guarantees employees' mental health.(16) The results of Shakerinia et al.'s study showed a significant negative relationship between nurses' job stress and resilience.(17)
Resilience is a dynamic process whereby individuals exhibit positive coping behaviours when faced with adversity(18). Increasing resilience is also an important goal in preventing and treating psychological injuries, ${ }^{(19)}$ so that one of the most important and fundamental principles of individuals' mental health is their resilience.(20)

Considering the inevitability of some stressors and the need to prevent the psychological and behavioural effects of stress, managers of organizations should employ measures to improve the QOWL and train coping strategies.(21) Therefore, considering the foregoing, the aim of the present study was to determine the effect of resilience training on QOWL and job stress of nurses in Emergency Department of Shahid Mohammadi Hospital in Bandar Abbas in 2018.

\section{METHODS}

\begin{tabular}{|c|c|}
\hline $\begin{array}{l}\text { Session } \\
\text { Number }\end{array}$ & Content of Resilience Training Sessions \\
\hline 1 & $\begin{array}{l}\text { Introduction to the general framework of the discussion: } \\
\text { - Definition of resilience } \\
\text { - Introducing characteristics of resilient people: } \\
\text { 1-Happiness 2-Wisdom and insight-3-Sense of humour-4-Empathy-5- } \\
\text { Intellectual competence-6-Purposeful life 7-Steadfastness } \\
\text { Solution: Identify unpleasant situations in life and increase adaptability } \\
\text { and tolerance in the individual domain }\end{array}$ \\
\hline 2 & $\begin{array}{c}\text { Objective: To familiarize with internal support factors } \\
\text { - The concept of optimism } \\
\text { - Source of Control } \\
\text { - To familiarize with stress and coping strategies } \\
\text { Solution: Recognize and emphasize talents and interests and desire to } \\
\text { use them }\end{array}$ \\
\hline 3 & $\begin{array}{c}\text { Objective: To get acquainted with external support factors } \\
\text { - Social support system } \\
\text { - Individual responsibility and acceptance of meaningful roles } \\
\text { - Cognitive restructuring and constructing a model of constructive } \\
\text { thinking (expressing the role of beliefs and thoughts in behaviours and } \\
\text { emotions and familiarity with cognitive errors) } \\
\text { Solution: Feeling a sense of being attached and valued and willing to } \\
\text { participate }\end{array}$ \\
\hline 4 & $\begin{array}{l}\text { Objective: To get acquainted with ways to create resilience } \\
\text { - Establish and maintain relationships with others } \\
\text { - Emphasize the importance of positive relationships with others and } \\
\text { attitude towards them } \\
\text { - Accept change } \\
\text { - To get acquainted with individual differences in perception, } \\
\text { emphasizing the importance of the role of thoughts and self-talk }\end{array}$ \\
\hline 5 & $\begin{array}{l}\text { Objective: To continue the ways of creating resilience } \\
\text { - Purposefulness and hoping for the future } \\
\text {-To act } \\
\text { - Familiarity with thinking styles and emphasis on the role of } \\
\text { optimism in resilience }\end{array}$ \\
\hline 6 & $\begin{array}{c}\text { Objective: To continue the ways of creating resilience } \\
\text { - Self-awareness } \\
\text {-Self-esteem } \\
\text { - Developing self-confidence }\end{array}$ \\
\hline 7 & $\begin{array}{c}\text { Objective: To continue the ways of creating resilience } \\
\text { - Self-care } \\
\text { - Framing the stressors }\end{array}$ \\
\hline 8 & $\begin{array}{l}\text { Objective: To continue the ways of creating resilience } \\
\text { - Search for meaning and seeking meaning in life } \\
\text { - Introducing a logotherapy approach and familiarity with Frankl's } \\
\text { experiences } \\
\text { - Emphasize the importance of giving meaning to immutable problems }\end{array}$ \\
\hline & Table 1. Content of Resilience Training Sessions \\
\hline
\end{tabular}

A total of 60 nurses working in the Emergency Department of Shahid Mohammadi Hospital in Bandar Abbas were included in the study using simple random sampling after the project's draft was approved, and confirmed by the Ethics Committee of Zabol University of Medical Sciences, and 
the necessary permissions were obtained. Inclusion criteria included consent to participate in the study, BA or MA in nursing, age range 25-60 years, absence of communication problems, absence of underlying diseases such as: diabetes, kidney failure, liver failure, and heart-lung failure, absence of known mental diseases, at least one year of work experience, and absence of pregnant women. They were selected and then randomly divided into two intervention and control groups based on drawing lots after providing necessary explanations about the aims and interventions of the research as well as completing written informed consent form.

Subjects in both groups completed demographic questionnaires, Walton Quality of Work Life Scale, and Nursing Stress Scale (NSS) developed by Gray-Taft and Anderson. The demographic questionnaire consists of 5 questions on age, gender, marital status, level of education, and years of work experience. Walton QOWL questionnaire consists of 35 questions in 8 dimensions of adequate and fair compensation, Safe and healthy environment, development of human capacities, growth and security, social integration, constitutionalism, the total life space, and social relevance. Each item is rated based on a 5-point Likert scale ranging from Very low (1), Low (2), Medium (3), High (4), and Very high (5). As a general rule, scores of 35-58 is defined as a low QWOl, 59-118 as a moderate QoWL, and above 118 as a high QoWL. Content validity and reliability were confirmed using Cronbach's alpha coefficients of $92 \%$ and $83 \%$, respectively. $(22,23)$ The researcher obtained Cronbach's alpha of 0.86 for Walton Quality of Work Quality of Life Questionnaire by studying 10 people, which indicates an appropriate. The Gray Taft \& Anderson Nursing Stress Scale (NSS) is a 34-item questionnaire on 7 dimensions of suffering and death of the patient, conflicts with physicians, lack of sufficient preparation, lack of support, conflict with other nurses, workload and uncertainty about treatment, and is scored based on 4-point Likert scale including: Never (0), Sometimes (1), Often (2), and Always (4). The lowest and highest scores are 34 and 102, respectively, with high scores indicating high stress and vice versa. The test-retest reliability of this questionnaire was reported 0.81 by Lee et al.(24) They also report the reliability range of 0.67 to 0.79 for the subscales of this questionnaire.(24) Lambert et al. also reported that the internal consistency of this scale was between 0.91-0.93. In the present study, the researcher obtained Cronbach's alpha of 0.89 for the Gray Taft and Anderson's NSS by studying individuals, which indicates an appropriate reliability.

In the intervention group, resilience training (Table 1) was delivered in the form 30-45-minute lecture for 8 sessions on a weekly basis in the conference hall of Shahid Mohammadi Hospital in Bandar Abbas. The educational content used was provided using credible scientific resources and approved by ten faculty members of Zabol School of Nursing and Midwifery. The control group did not receive any intervention during this period. One month after the intervention, the Walton Working Quality of Life Questionnaire and Gray Taft and Anderson's NSS were completed by the intervention and control groups again.

\section{RESULTS}

The findings showed that female participants made up for $60 \%$ and $53.3 \%$ of individuals in the intervention and control groups, respectively. Single participants also made up $80 \%$ and $73.3 \%$ of individuals in the intervention and control groups, respectively and there was no statistically significant difference between the two groups in terms of gender and marital status $(\mathrm{P}<0.05)$. Mean age of the intervention and control groups was 28.67 and 25.87 years, respectively. The mean of work experience was also in the intervention and control groups. There was no statistically significant difference between the two groups in terms of age and work experience $(\mathrm{P}<0.05)$.

According to RM-ANOVA test with Bonferroni ad-hoc test, there was no statistically significant difference between intervention and control groups in terms of post-intervention QoWL $(\mathrm{P}<0.05)$. RM-ANOVA test also showed no statistically significant difference between the two groups after the intervention in term of all QoWL dimensions except social integration and the total life space and constitutionalism in the organization $(\mathrm{P}<0.05)$ (Table 2$)$.

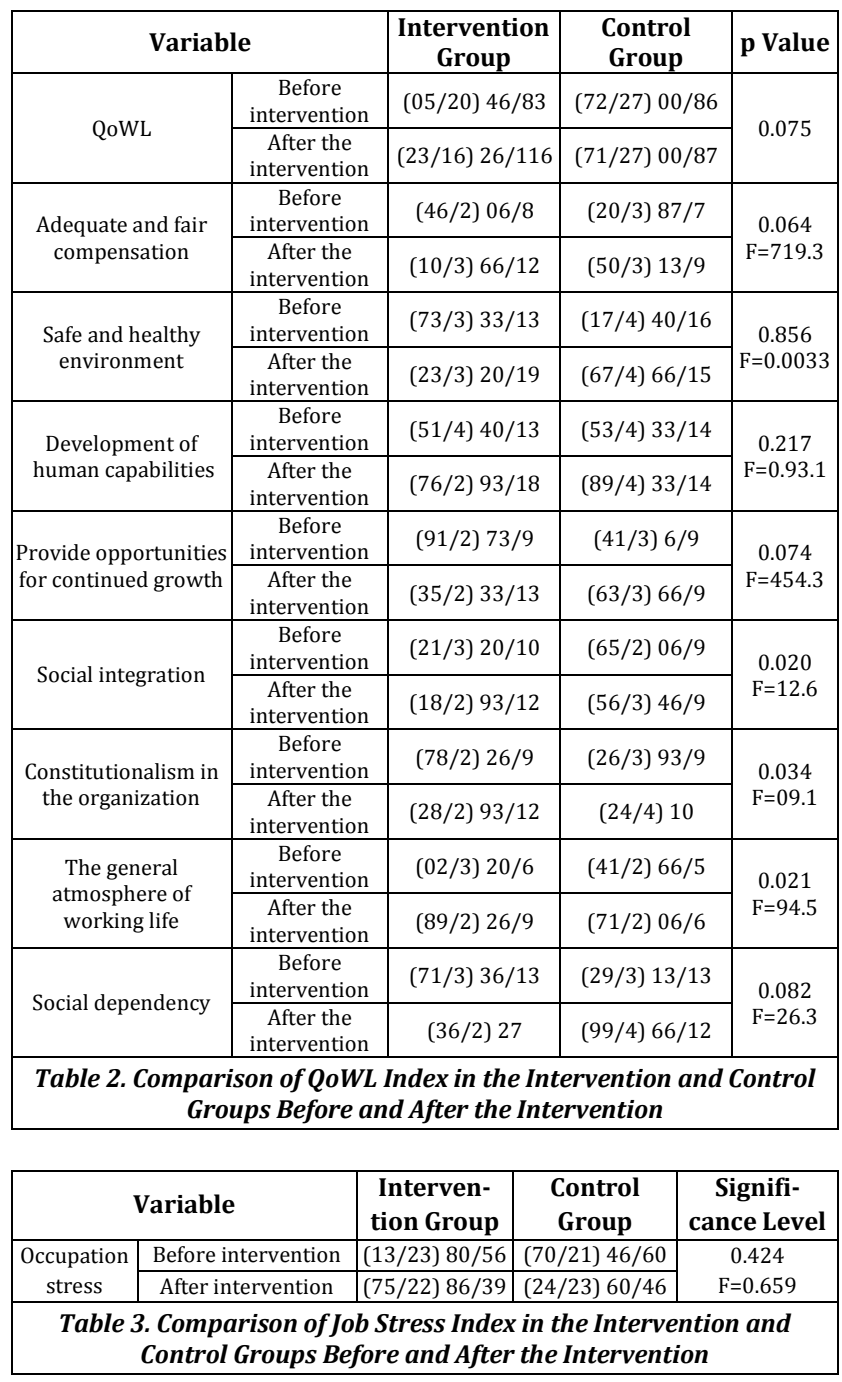

RM-ANOVA test showed no significant difference between the intervention and control groups after the intervention in terms of job stress ( $p>0.05)$ (Table 3$)$. 


\section{DISCUSSION}

The findings of the present research revealed no statistically significant difference between intervention and control groups after intervention in all QoWL dimension except social integration and the total life space and constitutionalism in organization ( $p>0.05)$. Latifzadeh et al. (2015) and (Miller et al. (2014) showed in their studies that resilience training improved nurses' QOL $(25,26)$. Leppin et al. also showed in a study that resilience training program in patients with chronic illness increased their QOL and adaptability.(27) Kaveh et al.'s study also showed that in resilience interventions in parents with mentally retarded children improved QOL components.(28) Since QOL has an impact on QoWL, ${ }^{(13)}$ none of the above studies were consistent with the results of the present study. The findings also revealed no statistically significant difference between the intervention and control groups after the intervention in terms of job stress (p0.05). Shakerinia et al. showed that resilience is a strong variable for reducing stress in nurses.(17) Sardarzadeh et al. also showed in their study that resilience intervention is effective on job stress among employees of Iranian Offshore Oil Company satellite project. (9)

A study by Momeni et al. (2014) showed that resilience training had a significant effect on the mental health of mothers of children with learning disorders. (29) Mehrabadi et al. (2010) showed that the resilience and immunization program improved job stress control among staffs of Tehran Payam Noor University.(30) Attention to related factors of QoL of nurses is very important. ${ }^{(31,32)}$ None of these studies were consistent with the results of the present study. The inconsistency between results of above studies with those of the present study may be due to the short duration of intervention and the study of QoWL and job stress 1 month after the intervention, as well as different working environment, organizational culture, and managerial approach, which may influence the results of the study.

One of the limitations of this study is that the nurses who participated in the study responded to the questions in the hospital setting and during a few minutes' rest, and thus lack of quiet atmosphere and suitable conditions can be considered as a limitation.

\section{CONCLUSIONS}

In organizations where employees enjoy an optimal QoWL, they put more effort in their organizations and such efforts lead to increased productivity. The results of this study show that resilience training can be used to improve QoWL in the indicators of social integration and the total life space and constitutionalism. Results of other indicators evaluated in other studies have also shown an improvement in QoWL Future studies with a larger sample size in the hospital by planners and consultants are recommended.

\section{ACKNOWLEDGEMENT}

This study is approved by the Ethics Committee (IR.ZBMU.REC.1397.147). It is also approved by Zabol University of Medical Sciences. The authors would like to express their gratitude to all the nurses who accompanied the researcher during the study. The authors are also grateful to the Vice Chancellor for Research of Zabol University of Medical Sciences for funding this project.

\section{REFERENCES}

[1] Nasiri-Ziba F, Bogheiri F. Mental health status of critical care nurses in teaching hospitals of Tehran. Iranian Journal Cardiovascular Nursing 2012;1(4):6-12. [Persian].

[2] Aghajani MJ. The professional burnout of nurses in different wards. Journal of Research in Nursing and Midwifery 2012;9(2):97-104. [Persian]

[3] Naderifar M, Tafreshi MZ, Ilkhani M, et al. Correlation between quality of life and adherence to treatment in hemodialysis patients. J Renal Inj Prev 2019;8(1):22-7.

[4] Adhikari DR, Gautam DK. Labor legislations for improving quality of work life in Nepal. Int J Law Manag 2010;52(1):40-53.

[5] Mohammadi MA, Mozaffari N, Dadkhah B, et al. Study of work-related quality of life of nurses in Ardabil province hospitals. Journal of Health and Care 2017;19(3):108-16. [Persian].

[6] Ghaleei A, Mohajeran B, Taajobi M, et al. Relationship between quality of work life and occupational stress in staff of Bu-ali Sina university of Hamadan, 2013. Pajouhan Scientific Journal 2015;13(4):60-6. [Persian].

[7] AbuAlRub RF. Job stress, job performance and social support among hospital nurses. Journal of Nursing Scholarship 2004;36(1):73-8.

[8] Jafari M, Houshmand HB, Maher A. Relationship of occupational stress and quality of work life with turnover intention among the nurses of public and private hospitals in selected cities of Guilan Province, Iran, in 2016. Journal of Health Research in Community 2017;3(3):12-24.[ Persian].

[9] Sardarzadeh F, Bagheri JM, Mashhadi FM. The role of resilience in reduction of job stress and job burnout among employees of satellite project of Iranian Offshore Oil Company. Shenakht Journal of Psychology and Psychiatry 2017;3(4):61-77. [Persian].

[10] Masoumy M, Tahmasebi R, Jalali M, et al. The Study of the relationship between Job stress and spiritual health of nurses working in Intensive care ward at Bushehr hospitals. Nursing Journal of the Vulnerable 2016;3(8):37-47. [Persian].

[11] Shareinia H, Khuniki F, Beydokhti TB, et al. Comparison between job stress among emergency department nurses with nurses of other departments. Quarterly Journal of Nursing Management 2018;6(3,4):48-56. [Persian].

[12] Jenaabadi H. Relationship between quality of life and quality of working life in staffs of Baharan psychiatry hospital, Zahedan. JQUMS 2013;17(1):61-8. [Persian].

[13] Janaabadi $H$, Nastiezaie N. Two effective factors in the staff's performance: quality of life and quality of working life. Zahedan J Res Med Sci 2012;13(1):9-10. [Persian]. 
[14] Irani ZB, Kulemarez BMJ, Hazari N. The effectiveness of resilience training program on improving nurses' quality of life. Quarterly Journal of Nursing Management 2017;5(3,4):9-18. [Persian].

[15] Mannix A, Margaret F. Optimism, resilience and leadership - 3 qualities to help move you toward greatness. 2011. http://enzinear ticles.com.

[16] Connor KM, Davidson JRT. Development of a new resilience scale: the Connor-Davidson Resilience Scale (CD-RISC). Depression and Anxiety 2003;18(2):76-82.

[17] Shakeriniya A, Mohammadpour M. Relationship between job stress and resilience with job Burnout in female nurses. Journal of Kermanshah University of Medical Sciences 2010;14(2):161-9. [Persian].

[18] Momeni K, Jalili Z, Mohseni R, et al. Efficacy of teaching resiliency on symptoms reduction on anxiety of adolescence with heart disease. J Clin Res Paramed Sci 2015;4(2):112-9. [Persian].

[19] Downey JA. Recommendations for fostering educational resilience in the classroom. Preventing School Failure 2008;53(1):56-64.

[20] Fathi D, Jamalabadi M. The study of examined mediating role of resiliency in the perception of academic support and academic buoyancy. Educ Strategy Med Sci 2017;10(4):263-9. [Persian].

[21] Khovnard B, Behbahani RN, Vise PP, et al. Relationship between job stress and quality of life in librarians Yazd Shahid Sadoughi University of Medical Sciences 2016;3(1):1-9. [Persian].

[22] Imani B, Arezo K, Mohammadi N. Association between quality of work life and occupational stresses in personnel of social security organization in Hamadan province, IR Iran. E3 J Med Res 2014;3(4):42-5. [Persian].

[23] Talasaz ZH, Saadoldin SN, Shakeri MT. Relationship between components of quality of work life with job satisfaction among midwives in Mashhad, 2014. J of Hayat 2015;21(1):56-67. [Persian].
[24] Lee MH, Holzemer WL, Faucett J. Psychometric evaluation of the Nursing Stress Scale (NSS) among Chinese nurses in Taiwan. Journal of Nursing Measurement 2007;15(2):133-44.

[25] Latifzadeh SH, Zarea K. Occupational stress and its related factors in nurses working in intensive care units of educational hospitals in Ahwaz, Iran. Journal of Novel Applied Sciences 2015;4(4):483-7. [Persian].

[26] Mealer M, Conrad D, Evans J, et al. Feasibility and acceptability of a resilience training program for intensive care unit nurses. American Journal of Critical Care 2014;23(6):e97-105.

[27] Leppin AL, Gionfriddo MR, Sood A, et al. The efficacy of resilience training programs: asystematic review protocol. Systematic Reviews 2014;3:20.

[28] Kaveh M, Alizadeh H, Delavar A, et al. Development of a resilience fostering program against stress and its impact on quality of life components in parents of children with mild intellectual disability. Journal of Exceptional Children 2011;11(2):119-40. [Persian].

[29] Momeni KH, Ebrahimi P, Hoseyniyan S. Improve mental health and quality of life through a resilient education program for Mothers of Children with Learning Disabilities. Education for women and family-Cultural 2014;8(26):39-64. [Persian].

[30] Mehrabadi F. Effectiveness of training of immunization against stress on the job burnout and resilience of employees of Payam Nour University of Tehran. Master thesis of general psychology. Payam Nour University 2010.

[31] Sheykhi M, Naderifar M, Firouzkohi M, et al. Effect of group logotherapy on death anxiety and occupational burnout of special wards nurses. Medical Science 2019;23(98):532-9.

[32] Naderifar M, Tafreshi MZ, Ilkhani M, et al. The outcomes of stress exposure in hemodialysis patients. J Renal Inj Prev 2017;6(4):275-281. 